\title{
Clinical Evidence of Association Between Type-2 Diabetes Mellitus and Hypothyroidism with Therapeutic Relevance-An Observational Study
}

\author{
Surampalli Gurunath ${ }^{*}$, Koyyada Arun², Amgoth Vamshi Krishna ${ }^{2}$, \\ Lekkala Kavya ${ }^{2}$ and Bathula Sridhar ${ }^{3}$
}

\begin{abstract}
${ }^{1}$ Department of Pharmacology, Vaagdevi Institute of Pharmaceutical Sciences, Warangal, Telangana, India; ${ }^{2}$ Department of Pharmacy Practice, Vaagdevi Institute of Pharmaceutical Sciences, Warangal, Telangana, India; ${ }^{3}$ Samraksha Diabetes, Thyroid and Endocrine, Superspeciality Hospital and Research Centre, Hanamkonda, Warangal, Telangana, India
\end{abstract}

\begin{abstract}
Background: Thyroid disorder and diabetes are associated endocrine disorders. Patients with either one of the disorders are at great risk of developing the other. The co-existence of type-2 diabetes mellitus (T2DM) and hypothyroidism with clinical evidence of effect on one another has not been reported until today.
\end{abstract}

Methods: This prospective study involved 702 patients. Each participant's blood sugar levels and thyroid profiles were statistically analyzed using unpaired $t$-test.

Results: The incidence of hypothyroidism and hyperthyroidism in diabetic patients was $20.2 \%$ and $1.71 \%$ respectively, while the incidence of type- 1 diabetes mellitus and T2DM in thyroid dysfunction patients was $0.56 \%$ and $19.03 \%$ respectively. The mean fasting blood sugars and postprandial blood sugar levels, triiodothyronine, thyroxine and Thyroid stimulating hormone levels of hypothyroid patients with T2DM (157.2 $\pm 4.779,231.7 \pm 6.291$, $1.123 \pm 0.065,8.643 \pm 1.519$ and $12.22 \pm 1.974)$ were not significantly different $(p>0.05)$ from those in T2DM patients with hypothyroidism $(153.6 \pm 7.181,236.6 \pm 9.504,1.091 \pm 0.013,7.971 \pm 1.024$ and $12.09 \pm 1.48)$. These findings were above the normal range (fasting blood sugar of 70-110 mg/dL; postprandial blood sugar of $>140$ $\mathrm{mg} / \mathrm{dL}$ ) thyroid stimulating hormone of $0.39-6.16 \mathrm{mIU} / \mathrm{L}$ in both type of patients, with the exception of triiodothyronine and thyroxine.

Conclusions: We report clinical evidence of association between hypothyroidism and diabetes mellitus with the highest incidence of hypothyroidism in T2DM with four major findings, suggesting regular thyroid and glycemic level evaluations for diabetic and hypothyroid patients respectively. Thus adjustment of dosage of levothyroxine is necessary, with prompt monitoring and reduction of the dose of insulin or oral hypoglycemic drugs to avoid hypoglycemia and its complications.

Keywords: Hypothyroidism; Hyperthyroidism; Type-1 DM; Type-2 DM; Endocrine disorders.

Abbreviations: FBS, fasting blood sugar; PLBS, postprandial blood sugar; T1DM, type-1 diabetes mellitus; T2DM, type-2 diabetes mellitus; T3, triiodothyronine; T4, thyroxine; TSH, thyroid stimulating hormone.

Received: March 18, 2019; Revised: July 05, 2019; Accepted: July 10, 2019

*Correspondence to: Surampalli-Gurunath, Department of Pharmacology, Vaagdevi Institute of Pharmaceutical Sciences, Warangal, Telangana, India. Tel: (+91) 9966555091; E-mail: s.gurunath1979@gmail.com

How to cite this article: Surampalli G, Koyyada A, Amgoth VK, Lekkala K, Bathula S. Clinical Evidence of Association Between Type-2 Diabetes Mellitus and Hypothyroidism with Therapeutic Relevance-An Observational Study. J Explor Res Pharmacol 2019;4(3):21-30. doi: 10.14218/JERP.2019.00001.

\section{Introduction}

Diabetes and thyroid disorders are the most common endocrine disorders seen in the general population. These two disorders are mutually associated and influence each other. In hypothyroidism, the metabolism of glucose is affected by several mechanisms, such as perturbed genetic expression of genes with physiological aberrations leading to impaired glucose utilization and disposal in muscles, overproduction of hepatic glucose output, and enhanced absorption of splanchnic glucose, ${ }^{1}$ thereby predisposing to diabetes mellitus. It is well established that hyper- and hypothyroidisms are associated with insulin resistance and are reported to be the 
Table 1. Percentage distribution of diabetic and thyroid patients

\begin{tabular}{lllll}
\hline & Type 1 DM (N = 25) & Type 2 DM (N = 325) & Hypothyroidism (N = 305) & Hyperthyroidism (N = 47) \\
\hline Male & $16(4.57 \%)$ & $159(45.42 \%)$ & $29(8.23 \%)$ & $05(1.4 \%)$ \\
Female & $09(2.57 \%)$ & $166(47.42 \%)$ & $276(78.4 \%)$ & $42(11.9 \%)$ \\
\hline
\end{tabular}

Abbreviation: DM, diabetes mellitus.

major cause of impaired glucose metabolism in type- 2 diabetes mellitus (T2DM) ${ }^{1}$ and worsen glycemic control. ${ }^{2}$

Among thyroid disorders, hypothyroidism is most common worldwide. It is commonly found to co-exist with type-1 diabetes mellitus (T1DM) and T2DM. It is thought to be the precipitating factor for hypoglycemia in diabetic patients. ${ }^{3}$ In addition the incidence of hypothyroidism increases with age and is more common in women. ${ }^{4}$

The prevalence of T2DM (about 90\%) is greater compared to that of T1DM (about 10\%) among the diabetes mellitus population due to increase in sedentary lifestyle and obesity. Also, the incidence of hypothyroidism is higher in T2DM patients than in T1DM patients. ${ }^{1}$ It is well known that thyroid hormones are insulin antagonists. Insulin has been found to inhibit the conversion of thyroxine (T4) to triiodothyronine (T3); thus, showing hypothyroidism in diabetic patients with significantly lower serum levels of T3 and T4 than in diabetic subjects without hypothyroidism. ${ }^{5}$ Moreover diabetic hypothyroid patients who were on metformin showed baseline reduction of thyroid stimulating hormone (TSH) level after 6 months. This shows the relation of antidiabetic therapy and risk of hypothyroidism. ${ }^{1}$

It has been three decades up to today to report that diabetes mellitus influences the assessment of thyrotoxicosis by falsely decreasing the blood levels of T4 and T3 with severely uncontrolled hyperglycemia. Hypothyroidism seems to ameliorate hyperglycemia. ${ }^{6}$ In a review published recently, Hage, et al. ${ }^{7}$ reported that diabetes mellitus and thyroid disorders tend to coexist in patients but none of the clinical studies have reported the diabetes mellitus and thyroid disorders interrelationship in a two-way fashion (particularly hypothyroidism $\leftrightarrow \mathrm{T} 2 \mathrm{DM}$ ). Our study has revealed, for the first time, the incidences of these disorders over prevalence with strong clinical evidence of association between hypothyroidism and T2DM and vice versa.

\section{Methods}

The study's protocol was approved prior to the start of the study by the Human Institutional Ethics Committee and all participants and/ or their parents or legally responsible persons provided informed consent. All procedures performed in our studies involving human participants were designed and carried out in accordance with the ethical standards of the institutional and/or national research committee and with the 1964 Helsinki Declaration and its later amendments, or comparable ethical standards.

This observational study was conducted in the North Telangana region. The study was conducted over a period of 6 months during 2017. The design was enabled to determine a) the incidence of T1DM and T2DM in patients with preexisting hypothyroidism and hyperthyroidism and b) the incidence of hypothyroidism and hyperthyroidism with pre-existing T1DM and T2DM. The diabetic patients enrolled were taking insulin and/or oral hypoglycemic agents and the hypothyroid patients were on levothyroxine $(25 / 50 / 75 / 88 / 100 / 125 \mathrm{mcg})$ therapy. A suitable data collection form was designed to collect, document and analyze the data. All the relevant and necessary data pertaining to blood glucose and thyroid profiles were collected from patients' laboratory reports. The total study population was 702 patients, of whom 350 patients had preexisting T1DM and T2DM and 352 patients had preexisting hypothyroidism and hyperthyroidism.

Based on the thyroid profile, hypothyroidism was considered when T3 and T4 levels were lesser and TSH levels were greater than the laboratory reference values. Hyperthyroidism was considered when T3 and T4 were greater and TSH levels were lesser than the laboratory reference values.

\section{Inclusion criteria}

1. Patients of either gender;

2. Males of 5-70 years old and females of 5-50 years old;

3. Patients with T1DM and T2DM, being at least 1 year from diagnosis;

4. Patients with hypothyroidism and hyperthyroidism, being at least 1 year from diagnosis.

\section{Exclusion criteria}

1. Post-menopausal women;

2. Patients with other co-morbidities (such as cardiac diseases and other endocrinology-related disorders) and their family history;

3. Chronic alcoholics and smokers.

\section{Statistical analysis}

Data were analyzed using GraphPad Prism software, version 6.0. Quantitative data were expressed using mean and standard deviation while qualitative data were expressed in frequency and percent. Quantitative data were analyzed using nonparametric Kruskal-Wallis test to compare between the two groups. The $p$ value was assumed to be significant at 0.05 or less.

\section{Results}

As stated above the total patients included in this study was 702 . Among them, 350 patients presented with predisposing diabetes (T1DM and T2DM). Out of those 350 patients, 25 had with T1DM and 325 had T2DM. Table 1 shows the number and percentage of distribution of diabetic patients. Also out of the 350 patients, $77(22 \%)$ presented with predisposing diabetes, affected with thyroid disorders (hypothyroidism and hyperthyroidism). The T2DM patients were more prone to be affected with thyroid dysfunction (with more hypothyroidism prevalence) than were the T1DM patients. The distribution of thyroid disorders in T1DM and T2DM is shown in Table 1. Based on our findings, we considered T2DM with and without hypothyroidism for statistical outcomes.

Out of the 702 total patients, the remaining 352 presented with 
Table 2. Distribution of thyroid disorders in diabetic patients and diabetes in thyroid patients respectively

\begin{tabular}{|c|c|c|c|c|c|c|c|c|}
\hline & \multicolumn{2}{|c|}{ Patients with T1DM } & \multicolumn{2}{|c|}{ Patients with T2DM } & \multicolumn{2}{|c|}{ Hypothyroidism patients } & \multicolumn{2}{|c|}{ Hyperthyroidism patients } \\
\hline & $\begin{array}{l}\text { Hypothyroidism } \\
(n=3)\end{array}$ & $\begin{array}{l}\text { Hyperthyroidism } \\
(n=0)\end{array}$ & $\begin{array}{l}\text { Hypothyroidism } \\
(n=68)\end{array}$ & $\begin{array}{l}\text { Hyperthyroidism } \\
(n=6)\end{array}$ & $\begin{array}{l}\text { Type-1 DM } \\
(\mathrm{n}=2)\end{array}$ & $\begin{array}{l}\text { Type-2 DM } \\
(n=65)\end{array}$ & $\begin{array}{l}\text { Type-1 DM } \\
(n=0)\end{array}$ & $\begin{array}{l}\text { Type-2 DM } \\
(\mathrm{n}=2)\end{array}$ \\
\hline Male & 00 & 00 & 13 & 00 & 00 & 11 & 00 & 00 \\
\hline Female & 03 & 00 & 55 & 06 & 02 & 54 & 00 & 02 \\
\hline
\end{tabular}

Abbreviations: T1DM, type-1 diabetes mellitus; T2DM, type-2 diabetes mellitus.

predisposing hypothyroidism $(n=305)$ and hyperthyroidism $(n=47)$ respectively. Hypothyroidism was found to be more affected in the general population than was hyperthyroidism. These patients were further screened for diabetes mellitus. Out of the 352 thyroid patients (both hypothyroidism and hyperthyroidism), 69 (19.6\%) were affected with diabetes. However, it was noticed that the patients with predisposing hypothyroidism were more prone to diabetes (more T2DM prevalence) than those with hyperthyroidism. Table 2 shows the number of thyroid distribution among diabetic patients.

To our surprise, thyroid disorder patients were more prone to developing T2DM than T1DM. The incidence of thyroid disorders (hypothyroidism and hyperthyroidism) in diabetes patients was found to be $22.5 \%$. The hypothyroidism incidence was significantly higher with $20.2 \%$ than the incidence of hyperthyroidism $(1.71 \%)$. Our findings showed that the incidence of thyroid disorders in diabetic patients was higher in females $(18.2 \%)$ than the males $(3.71 \%)$.

Further, our study included 352 thyroid patients (hypothyroidism and hyperthyroidism), among which the incidence of diabetes in known thyroid disorder patients $(n=352)$ was $19.6 \%$. Females with thyroid disorders were found to be $90.3 \%$ and among those incidences of getting diabetes was $15.9 \%$. T2DM incidence was greater in the thyroid patients than for T1DM.

Comparison of fasting blood sugar (FBS), postprandial blood sugar (PLBS) and thyroid hormones in T2DM patients with and without hypothyroidism

It was found that the mean FBS $(175.7 \pm 5.164 \mathrm{mg} / \mathrm{dL}, 153.6 \pm$ $7.181 \mathrm{mg} / \mathrm{dL}, p<0.0385)$ and PLBS $(261.4 \pm 5.971 \mathrm{mg} / \mathrm{dL}, 236.6$ $\pm 9.504 \mathrm{mg} / \mathrm{dL}, p<0.0481)$ of the diabetic patients without hypothyroidism were significantly higher when compared with those diabetic patients with hypothyroidism (Table 3 and Fig. 1a). Our findings showed significantly lowered levels of glucose in diabetic patients with hypothyroidism, as compared to those diabetic patients without hypothyroidism as reported by several studies. ${ }^{2,3}$ These results indicate hypoglycemia in T2DM patients under the influence of hypothyroidism.

The thyroid profile (T3, T4 and TSH) in the diabetic patients without hypothyroidism was compared with those diabetic patients with hypothyroidism. Table 3 and Figure $1 \mathrm{~b}$ show the comparison between the two groups containing T2DM with and without hypothyroidism. The statistical analysis using unpaired $t$-test showed that the T4, TSH but not T3 levels varied significantly ( $p$ $<0.05)$ between the groups. The mean T4 $(5.072 \pm 0.919 \mu \mathrm{g} / \mathrm{dL}$, $p<0.0231)$ and TSH $(8.12 \pm 1.73 \mathrm{mIU} / \mathrm{mL}, p<0.01)$ of T2DM without hypothyroidism were significantly less compared to those of T2DM with hypothyroidism $(7.971 \pm 1.024 \mu \mathrm{g} / \mathrm{dL}, 12.09 \pm 1.48$ $\mathrm{mIU} / \mathrm{mL})$. The mean values of T3 $(1.074 \pm 0.049 \mathrm{ng} / \mathrm{dL})$ of T2DM without hypothyroidism were not significant as compared to those of T2DM patients with hypothyroidism $(1.091 \pm 0.013 \mathrm{ng} / \mathrm{dL}, p>$ $0.654)$. These findings indicate that T2DM influences the thyroid profiles in hypothyroid patients with increasing levels of T4 and $\mathrm{TSH}$, because the patients were on treatment with metformin/insulin. The raised insulin levels inhibit conversion of T4 to T3.

Comparison of FBS, PLBS and thyroid hormones in hypothyroid patients with and without T2DM

It was found that the mean FBS $(157.2 \pm 4.779 \mathrm{mg} / \mathrm{dL}, 85.7 \pm$ $2.31 \mathrm{mg} / \mathrm{dL}, p<0.0014)$ and PLBS $(231.7 \pm 6.291 \mathrm{mg} / \mathrm{dL}, 123.7$ $\pm 4.5 \mathrm{mg} / \mathrm{dL}, p<0.0018$ ) of the hypothyroid patients with T2DM were significantly higher when compared with those hypothyroid patients without T2DM (Table 4 and Fig. 2a). These findings demonstrated that hypothyroidism in T2DM patients causes hyperglycemia with increased levels of FBS and PLBS. This is because the patients were on levothyroxine therapy, for which the increased thyroid hormones induces hepatic gluconeogenesis and increased absorption of glucose leading to increased sugar levels.

The thyroid profile (T3, T4 and TSH) in hypothyroid patients without diabetes was compared with predisposing thyroid patients with T2DM. Table 4 and Figure $2 \mathrm{~b}$ show the comparison between the two groups containing hypothyroid patients with and without T2DM. The statistical analysis using unpaired $t$-test showed that

Table 3. Comparison of FBS, PLBS and thyroid hormones in T2DM patients with and without hypothyroidism using unpaired $t$-test

\begin{tabular}{llll}
\hline Test & $\begin{array}{l}\text { Type-2 diabetics without hy- } \\
\text { pothyroidism }(\mathbf{n}=\mathbf{2 5 1})\end{array}$ & $\begin{array}{l}\text { Type-2 diabetics with hy- } \\
\text { pothyroidism }(\mathbf{n}=68)\end{array}$ & $\boldsymbol{p}$ value \\
\hline FBS $(\mathrm{mg} / \mathrm{dl})$ & $175.7 \pm 5.164$ & $153.6 \pm 7.181$ & $0.0385^{*}$ \\
PLBS $(\mathrm{mg} / \mathrm{dl})$ & $261.4 \pm 5.971$ & $236.6 \pm 9.504$ & $0.0481^{*}$ \\
TSH $(\mathrm{mlU} / \mathrm{ml})$ & $8.12 \pm 1.73$ & $12.09 \pm 1.48$ & $0.01^{*}$ \\
T3 $(\mathrm{ng} / \mathrm{dl})$ & $1.074 \pm 0.049$ & $1.091 \pm 0.013$ & $0.654 \#$ \\
T4 $(\mu \mathrm{g} / \mathrm{dl})$ & $5.072 \pm 0.919$ & $7.971 \pm 1.024$ & $0.0231^{*}$ \\
\hline
\end{tabular}

Data is expressed as mean \pm SEM with * showing significant values $p<0.05$, \# insignificant values. Abbreviations: FBS, fasting blood sugar; PLBS, postprandial blood sugar; T2DM, type-2 diabetes mellitus. 


\section{a}

Comparison of FBS and PLBS in T2DM patients with and without hypothyroidism using unpaired t-test

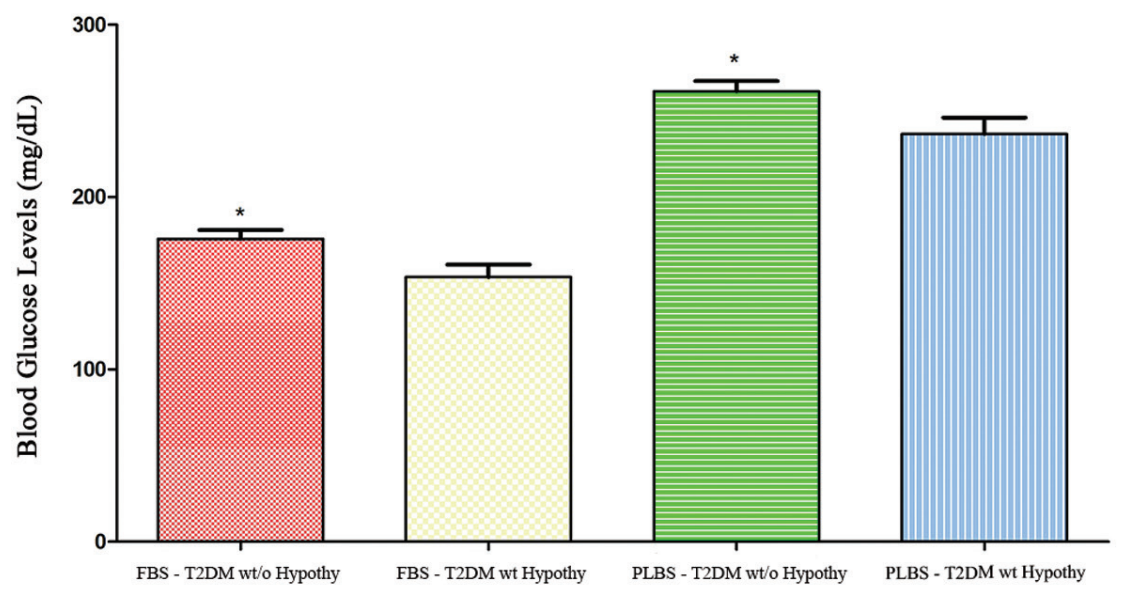

b Comparison of thyroid hormones profiles in T2DM patients with
and without hypothyroidism using unpaired t-test

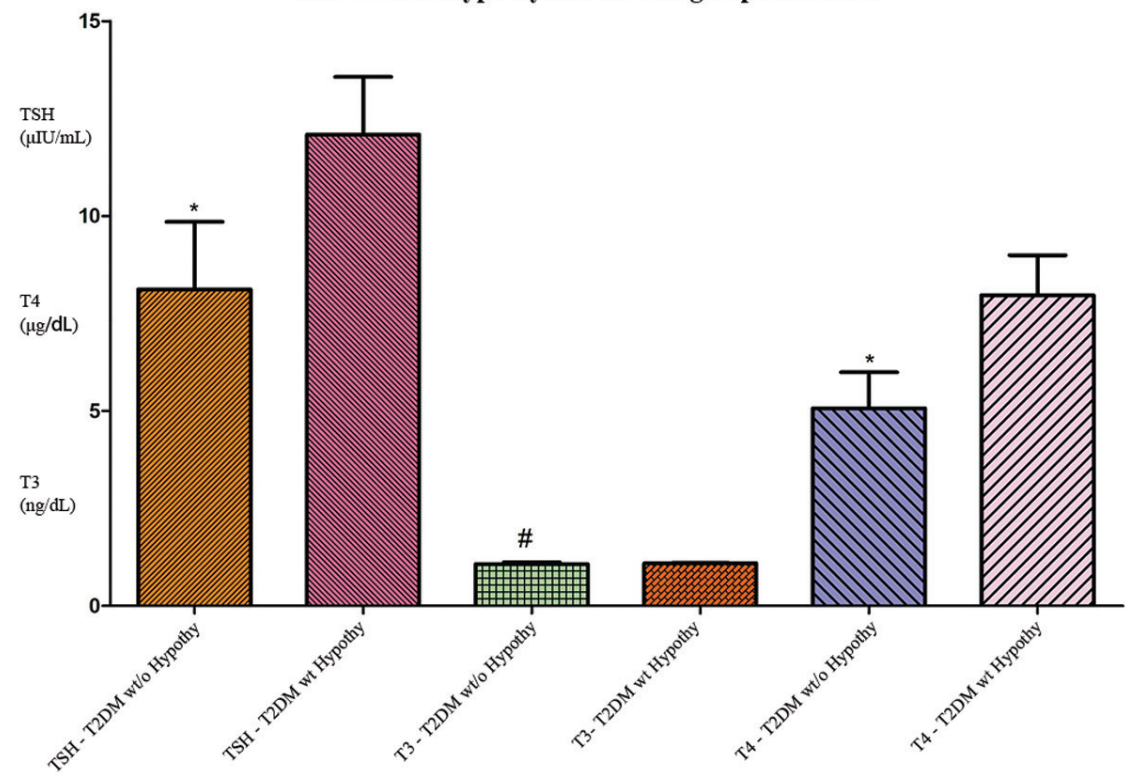

Fig. 1. (a) Comparison of FBS and PLBS in T2DM patients with and without hypothyroidism using unpaired t-test; (b) Comparison of thyroid hormone profiles in T2DM patients with and without hypothyroidism using unpaired $t$-test. FBS, fasting blood sugar; Hypothy, Hypothyrodism; PLBS, postprandial blood sugar; T2DM, type-2 diabetes mellitus. wt/o, without; wt, with; * means statistically significant at $P<0.05$, \#means statistically insignificant.

the T4 and TSH but not T3 levels varied significantly $(p<0.05)$ between the groups. The mean TSH levels $(12.22 \pm 1.974 \mathrm{mIU} / \mathrm{mL}$, $p<0.0016$ ) of thyroid patients without diabetes were found to be significantly less than those for the hypothyroid patients with diabetes $(27.95 \pm 2.574 \mathrm{mIU} / \mathrm{mL})$ whereas T4 $(8.643 \pm 1.519 \mu \mathrm{g} / \mathrm{dL}, p<$ 0.0287 ) of hypothyroid patients without diabetes were significantly more compared to that of predisposing hypothyroid patients having diabetes $(6.675 \pm 0.208 \mu \mathrm{g} / \mathrm{dL})$. The mean values of $\mathrm{T} 3(1.321 \pm$ $0.074 \mathrm{ng} / \mathrm{dL}, 1.123 \pm 0.065 \mathrm{ng} / \mathrm{dL}$ ) of thyroid patients with diabetes were not significantly different $(p<0.5389)$ from those of the group of thyroid patients without diabetes. These results demonstrate that hypothyroidism in T2DM patients is related to decreased levels of TSH when compared to hypothyroid patients without diabetes. However, T4 levels were raised in the hypothyroid patients with T2DM, without any changes in the levels of T3.

Comparison of FBS, PLBS and thyroid hormones in T2DM patients without hypothyroidism and hypothyroid patients without T2DM

It was found that the mean FBS $(175.7 \pm 5.164 \mathrm{mg} / \mathrm{dL}, 85.7 \pm 2.31$ $\mathrm{mg} / \mathrm{dL}, p<0.01)$ and PLBS $(261.4 \pm 5.971 \mathrm{mg} / \mathrm{dL}, 123.7 \pm 4.5$ $\mathrm{mg} / \mathrm{dL}, p<0.01$ ) of the hypothyroid patients without T2DM was 
Table 4. Comparison of FBS, PLBS and thyroid hormones in hypothyroid patients with and without T2DM using unpaired $t$-test

\begin{tabular}{llll}
\hline Test & $\begin{array}{l}\text { Hypothyroidism without } \\
\text { Type-2 DM }(\mathbf{n}=\mathbf{2 3 6})\end{array}$ & $\begin{array}{l}\text { Hypothyroidism with } \\
\text { Type-2 DM }(\mathbf{n}=65)\end{array}$ & $p$ value \\
\hline FBS $(\mathrm{mg} / \mathrm{dl})$ & $85.7 \pm 2.31$ & $157.2 \pm 4.779$ & $0.0014^{*}$ \\
PLBS $(\mathrm{mg} / \mathrm{dl})$ & $123.7 \pm 4.5$ & $231.7 \pm 6.291$ & $0.0018^{*}$ \\
TSH $(\mathrm{mlU} / \mathrm{ml})$ & $27.95 \pm 2.574$ & $12.22 \pm 1.974$ & $0.0016^{* *}$ \\
T3 $(\mathrm{ng} / \mathrm{dl})$ & $1.321 \pm 0.074$ & $1.123 \pm 0.065$ & $0.0538 \#$ \\
T4 $(\mu \mathrm{g} / \mathrm{dl})$ & $6.675 \pm 0.208$ & $8.643 \pm 1.519$ & $0.0287^{*}$ \\
\hline
\end{tabular}

Data is expressed as mean \pm SEM with $*$ significant, ${ }^{* *}$ highly significant values $p<0.05$, \# insignificant values. Abbreviations: FBS, fasting blood sugar; PLBS, postprandial blood sugar; T2DM, type-2 diabetes mellitus.

significantly less when compared with those of the T2DM patients without hypothyroidism (Table 5 and Fig. 3a). These findings illustrated that hypothyroidism influences FBS and PLBS levels in the absence of T2DM.

The thyroid profile (T3, T4 and TSH) in T2DM without hypothyroidism was compared to that of patients with predisposing hypothyroid and without T2DM. Table 5 and Figure 3 b show the comparison between the two groups containing T2DM without hypothyroidism and hypothyroid patients without T2DM. The statistical analysis using unpaired $t$-test showed that the T4 and TSH but not T3 levels varied significantly $(p<0.05)$ between the groups. The mean T4 $(6.675 \pm 0.208 \mu \mathrm{g} / \mathrm{dL}, p<0.013)$ and TSH $(27.95 \pm 2.574 \mathrm{mIU} / \mathrm{mL}, p<0.0034)$ levels of hypothyroid patients without diabetes were significantly greater compared to those of patients with predisposing T2DM without hypothyroidism $(5.072 \pm 0.919 \mu \mathrm{g} / \mathrm{dL}, 8.12 \pm 1.73 \mathrm{mIU} / \mathrm{mL})$. The mean values of T3 $(1.074 \pm 0.049 \mathrm{ng} / \mathrm{dL})$ of T2DM patients without hypothyroidism were not significantly different $(p<0.571)$ as compared to those of the hypothyroid patients without T2DM $(1.321 \pm 0.074 \mathrm{ng} / \mathrm{dL})$. These findings indicated that T2DM also influences the thyroid profiles in the absence of hypothyroidism, but at a subclinical level.

Comparison of FBS, PLBS and thyroid hormones in T2DM patients with hypothyroidism and hypothyroid patients with T2DM using unpaired t-test

It was found that the mean FBS $(157.2 \pm 4.779 \mathrm{mg} / \mathrm{dL}, 153.6 \pm$ $7.181 \mathrm{mg} / \mathrm{dL}, p>0.05)$ and PLBS $(231.7 \pm 6.291 \mathrm{mg} / \mathrm{dL}, 236.6$ $\pm 9.504 \mathrm{mg} / \mathrm{dL}, p>0.05)$ of the hypothyroid patients with T2DM were not significantly different when compared to those of T2DM patients with hypothyroidism (Table 6 and Fig. 4a). These findings indicated that either T2DM or hypothyroidism were equally effective on each other with reference to glycemic levels.

The thyroid profile (T3, T4 and TSH) in T2DM with hypothyroidism was compared with that of predisposing hypothyroid patients with T2DM. Table 6 and Figure 4b shows the comparison between the two groups containing T2DM patients with hypothyroidism and hypothyroid patients with T2DM. The statistical analysis using unpaired $t$-test showed that the T4, TSH and T3 levels were not varied significantly $(p>0.05)$ between the groups. The mean values of T3 $(1.091 \pm 0.013 \mathrm{ng} / \mathrm{dL}, 1.123 \pm 0.065 \mathrm{ng} / \mathrm{dL}$, $p>0.075) ; \mathrm{T} 4(7.971 \pm 1.024 \mu \mathrm{g} / \mathrm{dL}, 8.643 \pm 1.519 \mu \mathrm{g} / \mathrm{dL}, p>$ $0.057)$ and TSH $(12.09 \pm 1.48 \mathrm{mIU} / \mathrm{mL}, 12.22 \pm 1.974 \mathrm{mIU} / \mathrm{mL}, p$ $>0.067$ ) of T2DM patients with hypothyroidism were not significantly different when compared to those of hypothyroid patients with T2DM. These findings showed that either T2DM or hypo- thyroidism were equally influencing on one another with respect to thyroid profile.

\section{Discussion}

This study was done with the purpose of evaluating the incidence of thyroid disorders (hypothyroidism and hyperthyroidism) in diabetes mellitus and vice versa to evaluate their effects on one another. Our study provided impressive results with significant correlation between hypothyroidism and T2DM and vice versa. Several clinical studies in endocrinology showed the relationship between thyroid disorders and diabetes mellitus, but none have shown their interrelationship in a two-way fashion (hypothyroidism $\leftrightarrow \mathrm{T} 2 \mathrm{DM}$ ). Our study revealed the first of its kind in reporting novel clinical evidence of incidence and the interrelationship between hypothyroidism and T2DM and vice versa. In diabetic patients the incidence of hypothyroidism was found to be greater compared to the patients with hyperthyroidism, whereas in thyroid dysfunction patients incidence of T2DM is greater compared to the patients with T1DM. Therefore, hypothyroidism in T2DM and T2DM in hypothyroidism were taken into consideration for this study.

This is a unique clinical study as a clinical evidence to be new of its kinds showing an association between T2DM and hypothyroidism and vice versa, since no such reports are available until today.

The mean FBS and PLBS levels of T2DM patients without hypothyroidism was significantly higher than those of the T2DM patients with hypothyroidism. It is well-known, that hypoglycemia occurs in a greater amount of diabetic hypothyroid patients than in diabetic patients without hypothyroidism. The significant factors that contribute to hypoglycemia are reduced insulin clearance, slow gastric emptying and decreased intestinal absorption of glucose. Further, biochemical effects like reduced gluconeogenesis, impaired glycogenolysis and reduced glucagon secretion prevent recovery from hypoglycaemia. ${ }^{8,9}$ Additionally, the hepatic glucose output is decreased followed by prolonged insulin half-life. Therefore, the doses of exogenous insulin may have to be decreased to avoid hypoglycaemia. ${ }^{10-12}$ On the other hand, hypothyroid patients with T2DM show significantly increased T4 and decreased T3 and TSH levels when compared to hypothyroid patients without T2DM; this agrees with the previously reported observations of insulin enhancing the levels of T4 while suppressing the levels of T3 by inhibiting hepatic conversion of T4 to T3. ${ }^{13}$ On the other hand, diabetic hypothyroid patients showed increased T4 and TSH levels when compared with diabetic patients without hypothyroidism. These findings agree with another study, in which 
Comparison of FBS and PLBS in hypothyroid patients with
and without T2DM using unpaired t-test
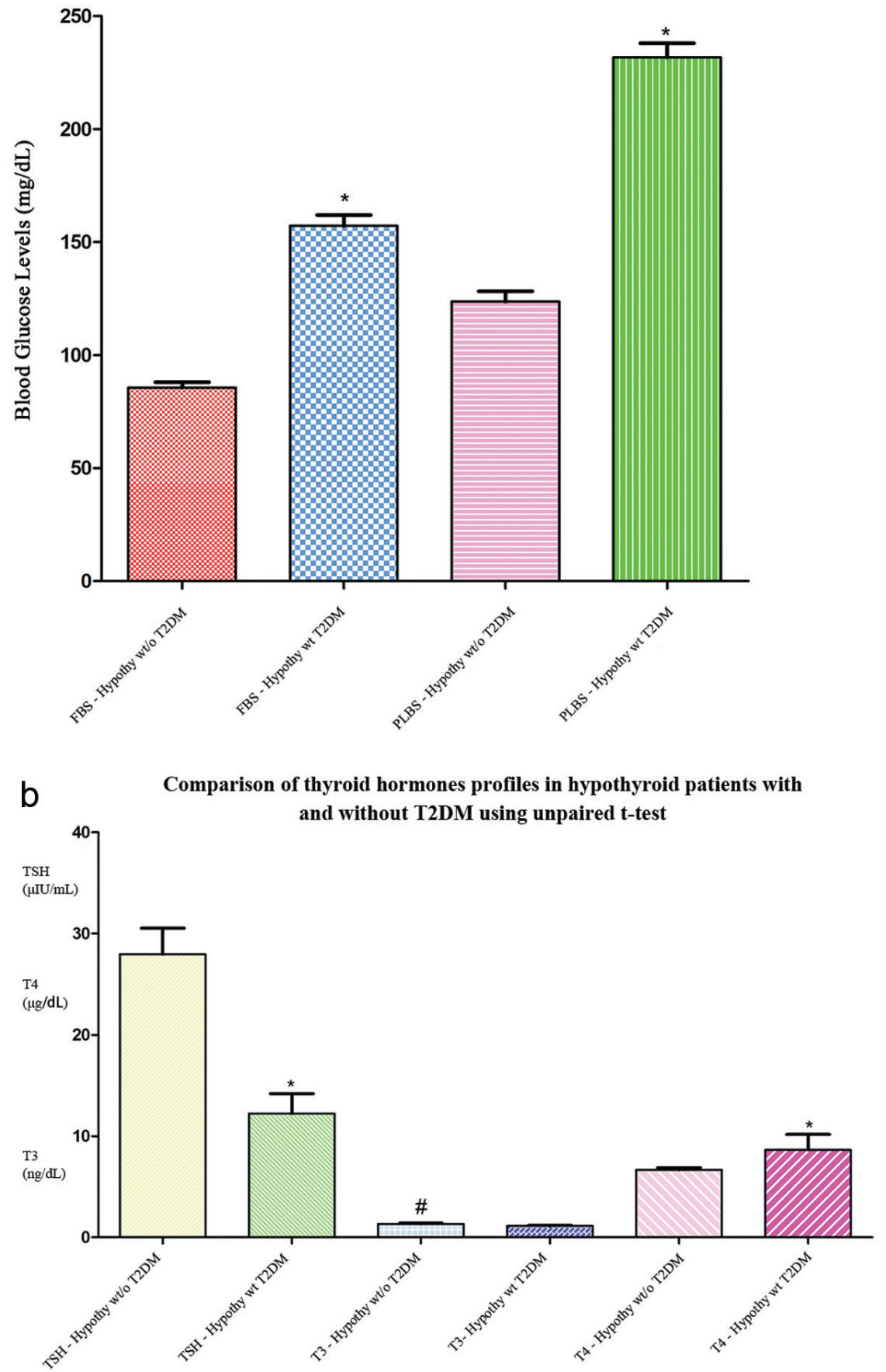

Fig. 2. (a) Comparison of FBS and PLBS in hypothyroid patients with and without T2DM using unpaired $t$-test; (b) Comparison of thyroid hormone profiles in hypothyroid patients with and without T2DM using unpaired $t$-test. FBS, fasting blood sugar; Hypothy, Hypothyrodism; PLBS, postprandial blood sugar; T2DM, type-2 diabetes mellitus. wt/o, without; wt, with; *means statistically significant at $P<0.05$, \#means statistically insignificant.

raised serum TSH levels were observed in T2DM patients suffering from hypothyroidism. ${ }^{8}$ The main findings of our study are that our diabetic patients were more prone to thyroid disorders than were our thyroid patients to getting diabetes, with female patients being more at risk.
Upon comparing the mean FBS and PLBS levels of T2DM without hypothyroidism and hypothyroidism without T2DM, we found hypoglycemia in hypothyroid patients without T2DM. Hypothyroidism is linked with various hormonal, biochemical and nervous system abnormalities which may contribute to hy- 
Table 5. Comparison of FBS, PLBS and thyroid hormones in T2DM patients without hypothyroidism and hypothyroid patients without T2DM using unpaired $t$-test

\begin{tabular}{llll}
\hline Test & $\begin{array}{l}\text { Type-2 diabetics without hy- } \\
\text { pothyroidism }(\mathbf{n}=\mathbf{2 5 1})\end{array}$ & $\begin{array}{l}\text { Hypothyroidism without } \\
\text { Type-2 DM }(\mathbf{n}=\mathbf{2 3 6})\end{array}$ & $p$ value \\
\hline FBS $(\mathrm{mg} / \mathrm{dl})$ & $175.7 \pm 5.164$ & $85.7 \pm 2.31$ & $0.01^{*}$ \\
PLBS $(\mathrm{mg} / \mathrm{dl})$ & $261.4 \pm 5.971$ & $123.7 \pm 4.5$ & $0.01^{*}$ \\
TSH $(\mathrm{mlU} / \mathrm{ml})$ & $8.12 \pm 1.73$ & $27.95 \pm 2.574$ & $0.0034^{* *}$ \\
Total T3 $(\mathrm{ng} / \mathrm{dl})$ & $1.074 \pm 0.049$ & $1.321 \pm 0.074$ & $0.571 \#$ \\
Total T4 $(\mu \mathrm{g} / \mathrm{dl})$ & $5.072 \pm 0.919$ & $6.675 \pm 0.208$ & $0.432 \#$ \\
\hline
\end{tabular}

Data is expressed as mean \pm SEM with * significant at $p<0.05$, \# insignificant values. Abbreviations: FBS, fasting blood sugar; PLBS, postprandial blood sugar; T2DM, type-2 diabetes mellitus.

poglycemia. Hypothyroid patients have relative adrenal insufficiency, even if the patients do not have primary adrenal failure. There is a blunted hypothalamo-pituitary-adrenal response to hypoglycemia in hypothyroid patients. ${ }^{14,15}$ However, significant changes in the thyroid profile in terms of TSH levels were noticed in our hypothyroid patients without T2DM than the T2DM patients without hypothyroidism. Agreeing with findings from other studies, T2DM showed prominent effect on thyroid hormones with the prevalence of thyroid disorders in patients of T2DM. ${ }^{7,8,10,11,16-18}$

A cross-sectional study reported high incidence of thyroid dysfunction $(28 \%)$ in T2DM patients, with subclinical hypothyroidism $(18.8 \%)$ as the most common thyroid disorder. ${ }^{19}$ In addition, Ravishankar et al. ${ }^{20}$ reported the prevalence of thyroid disorders in T2DM as $29 \%,{ }^{20}$ in agreement with the results of Unnikrishnan and colleagues (overall prevalence of hypothyroidism begin $10.95 \%(n=587)$ of which $3.47 \%$ were previously undetected and $7.48 \%$ were self-reported cases). ${ }^{21}$ Further, Prasad et al, ${ }^{22}$ reported the $23 \%$ prevalence of hypothyroidism and $10 \%$ of hyperthyroidism with marked increase in level of TSH among diabetic patients with hypothyroidism with significant change in level of T3, T4 and TSH among cases and controls. ${ }^{22}$ The published results from Rai et al. ${ }^{23}$ further supported our findings with decreased serum T3 and T4 and increased TSH levels in T2 diabetics without any complications. ${ }^{24,25}$

Further support for our findings is found among the recent studies that have shown that diabetic patients with nephropathy may be complicated by primary hypothyroidism. But, our study did not include T2DM patients with complications, which underlies the limitations of our findings for suggesting only minor changes in thyroid profiles in T2DM patients. Therefore further studies should be planned to evaluate the diabetic population regarding hypothyroidism as to whether it is clinical or sub-clinical.

Upon comparison of the mean FBS and PLBS levels of disorders (T2DM with hypothyroidism and hypothyroidism with T2DM), no significant changes in glycemic levels were noticed in hypothyroid diabetic patients when compared to diabetic hypothyroid patients. But, it was observed that FBS, PLBS and TSH levels were above the normal range in both the groups of patients and $\mathrm{TSH}$, except T3 and T4. These findings indicate that either of the disorders is equally dominant on one another when normal ranges of glycemic and thyroid levels are taken into consideration. Therefore neither T2DM with hypothyroidism nor hypothyroidism with T2DM was found to be significant; hence, both of them are to be controlled and monitored strictly as co-existence of each other significantly influencing one another and relating to the normal values of glycemic and thyroid levels. Our findings stand out to be novel for endocrinologists and other researchers with prominent clinical evidence showing the association between T2DM and hypothyroidism and vice versa.

Our study findings signify clinical evidence of association between T2DM and hypothyroidism. The incidence of hypothyroidism was decreasing the levels of blood glucose in pre-existing diabetic patients, so that the dose of insulin or oral hypoglycemic drugs should be reduced to avoid hypoglycemia and its complications. On the other hand, it was observed that pre-existing hypothyroidism in diabetic patients, led to decreased T3 and TSH levels thus necessitating the adjustment of dosage of levothyroxine with prompt monitoring.

\section{Conclusions}

Our study showed that a significant interrelationship co-exists between hypothyroidism and diabetes mellitus. T2DM patients were more prone to be affected with thyroid dysfunction (hypothyroidism) than T1DM patients. The incidence of thyroid disorder in diabetes patients was significantly higher than the incidence of diabetes in thyroid disorder patients. On the other hand the patients with predisposing hypothyroidism were more prone to diabetes (T2DM) than those with hyperthyroidism. Hence, our study concluded four major findings:

1. Comparison of FBS, PLBS and thyroid hormones in T2DM patients with and without hypothyroidism significantly lowered the glucose levels in diabetic patients, with hypothyroidism demonstrating hypoglycemia with increasing levels of T4 and TSH.

2. Comparison of FBS, PLBS and thyroid hormones in hypothyroid patients with and without T2DM demonstrated increased levels of FBS and PLBS, thus signifying hyperglycemia with decreased levels of TSH and raised T4 levels without any changes in the levels of T3.

3. Comparison of FBS, PLBS and thyroid hormones in T2DM patients without hypothyroidism and hypothyroid patients without T2DM demonstrated that hypothyroidism influenced FBS and PLBS levels in the absence of T2DM. Likewise, T2DM also influenced the thyroid profiles in the absence of hypothyroidism but at a subclinical level.

4. Comparison of FBS, PLBS and thyroid hormones in T2DM patients with hypothyroidism and hypothyroid patients with T2DM illustrated the coexistence of each other (i.e. T2DM with hypothyroidism or hypothyroidism with T2DM significantly influences one another relating to the normal values of glycemic and thyroid levels). Therefore, neither T2DM with hypothyroidism nor hypothyroidism with T2DM was found 


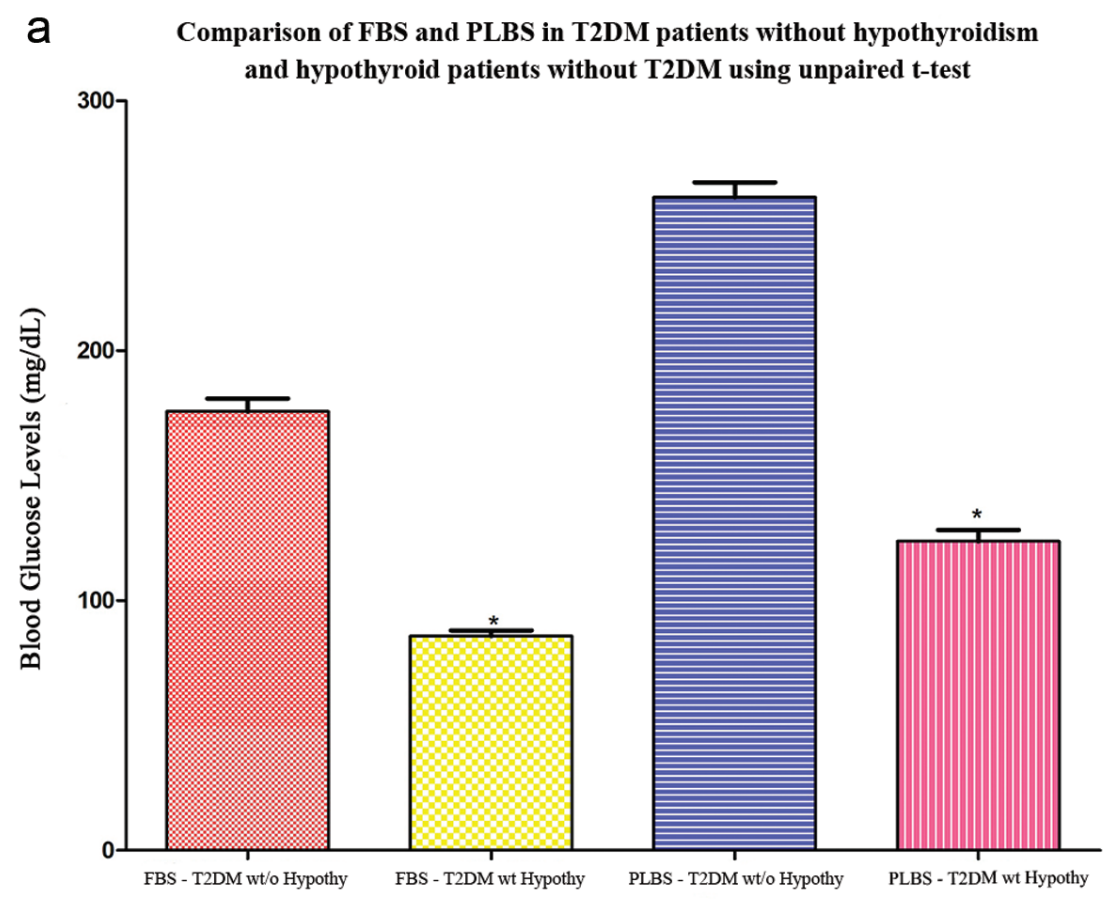

b Comparison of thyroid hormones profiles in T2DM patients without hypothyroidism

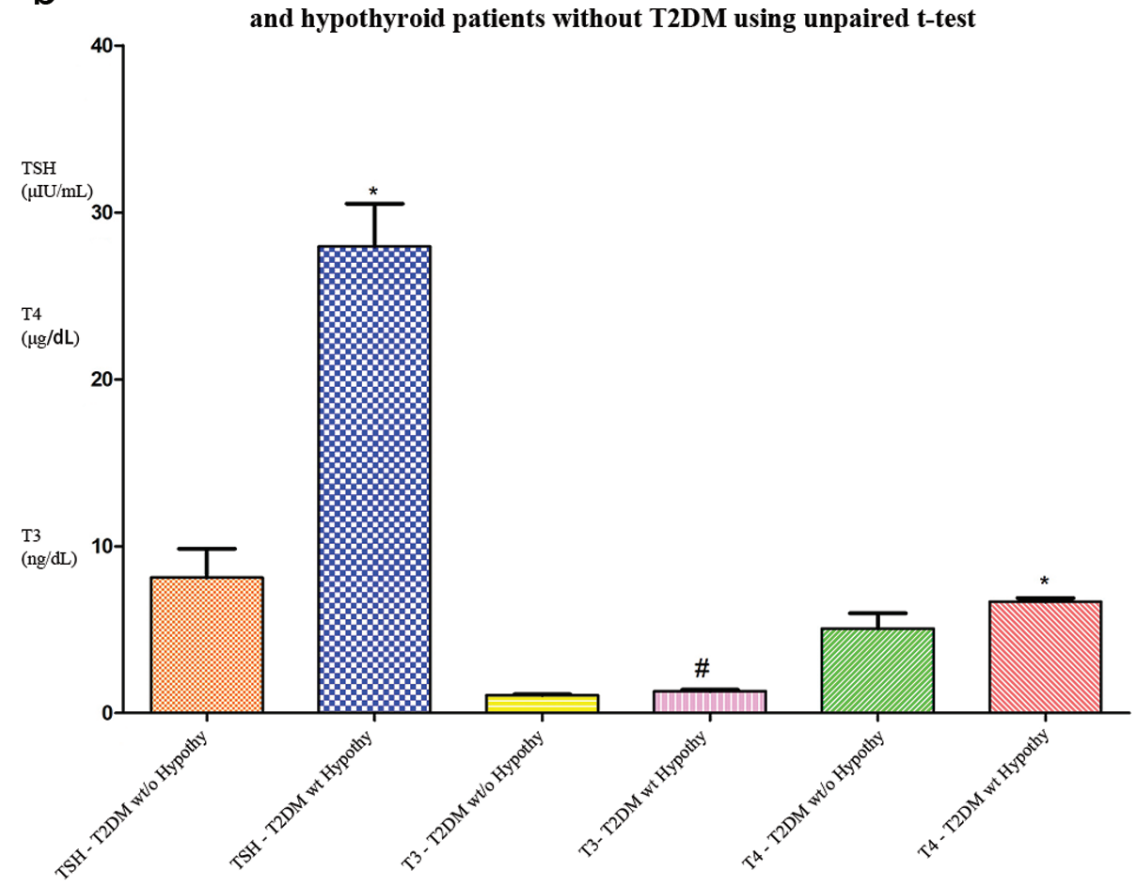

Fig. 3. (a) Comparison of FBS and PLBS in T2DM patients without hypothyroidism and hypothyroid patients without T2DM using unpaired $t$-test; (b) Comparison of thyroid hormone profiles in T2DM patients without hypothyroidism and hypothyroid patients without T2DM using unpaired $t$-test. FBS, fasting blood sugar; Hypothy, Hypothyrodism; PLBS, postprandial blood sugar; TSH, Thyroid stimulating hormone; T2DM, type-2 diabetes mellitus; T3, triiodothyronine; T4, thyroxine. wt/o, without; wt, with; *means statistically significant at $P<0.05$, \#means statistically insignificant.

to be significant, suggesting that both should be controlled and monitored strictly when co-existing.

Therefore, it is advisable to perform the regular thyroid evaluation for those patients who are diabetic and vice versa to avoid the complications and poor management of either. By early diagnosis and therapeutic intervention, one can improve the health status of the patient. Furthermore, related studies must be conducted in the near future to gain a better understanding of the underlying mecha- 
Table 6. Comparison of FBS, PLBS and thyroid hormones in T2DM patients with hypothyroidism and hypothyroid patients with T2DM using unpaired t-test

\begin{tabular}{llll}
\hline Test & $\begin{array}{l}\text { Type-2 diabetics with hy- } \\
\text { pothyroidism }(\mathbf{n}=68)\end{array}$ & $\begin{array}{l}\text { Hypothyroidism with } \\
\text { Type-2 DM }(\mathbf{n}=65)\end{array}$ & $p$ value \\
\hline FBS & $153.6 \pm 7.181$ & $157.2 \pm 4.779$ & $0.347 \#$ \\
PLBS & $236.6 \pm 9.504$ & $231.7 \pm 6.291$ & $0.231 \#$ \\
TSH & $12.09 \pm 1.48$ & $12.22 \pm 1.974$ & $0.675 \#$ \\
T3 & $1.091 \pm 0.013$ & $1.123 \pm 0.065$ & $0.758 \#$ \\
T4 & $7.971 \pm 1.024$ & $8.643 \pm 1.519$ & $0.579 \#$ \\
\hline
\end{tabular}

Data is expressed as mean \pm SEM with \# insignificant values $p>0.05$. Abbreviations: FBS, fasting blood sugar; PLBS, postprandial blood sugar; T2DM, type-2 diabetes mellitus.
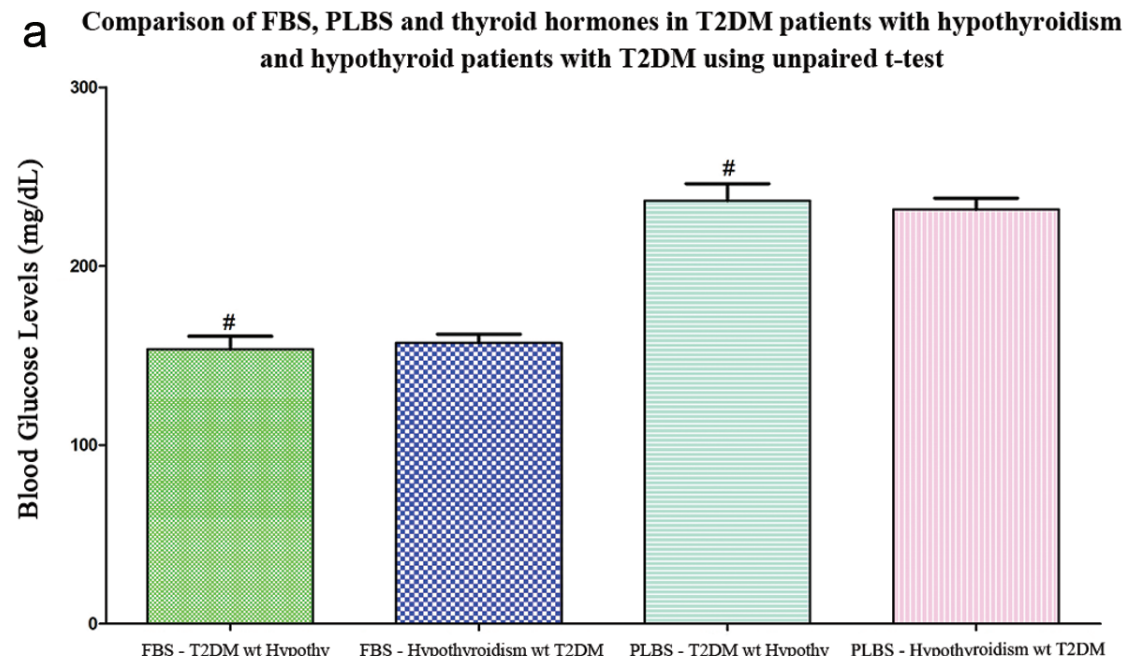

b Comparison of thyroid hormones profiles in T2DM patients with hypothyroidism and hypothyroid patients with T2DM using unpaired t-test

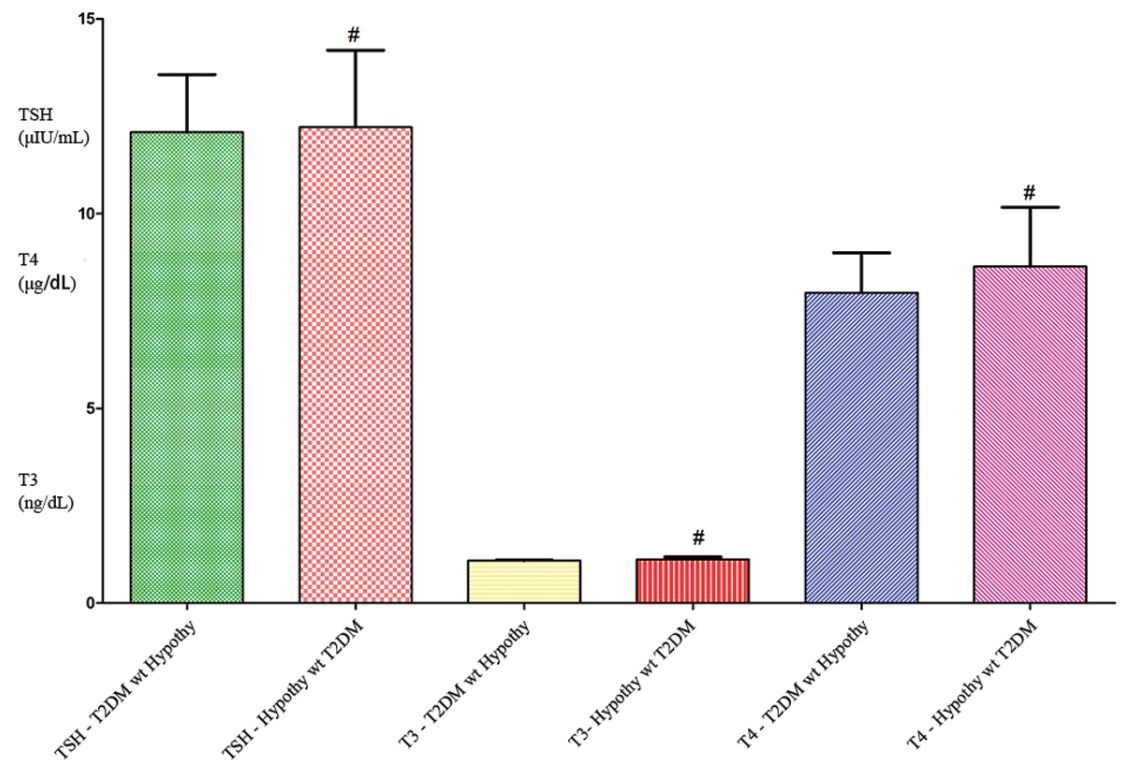

Fig. 4. (a) Comparison of FBS and PLBS in T2DM patients with hypothyroidism and hypothyroid patients with T2DM using unpaired $t$-test; (b) Comparison of thyroid hormones profiles in T2DM patients with hypothyroidism and hypothyroid patients with T2DM using unpaired $t$-test. FBS, fasting blood sugar PLBS, postprandial blood sugar; TSH, Thyroid stimulating hormone; T2DM, type-2 diabetes mellitus. T3, triiodothyronine; T4, thyroxine. wt/o, without; wt, with; \#means statistically insignificant at $P>0.05$. 
J Explor Res Pharmacol

nisms for the observed association described herein.

Future research directions, prospectives, and predictions

Further clinical studies are under way, for the study of various risk factors and their mechanisms influencing the association of T2DM and hypothyroidism, and vice-versa.

\section{Acknowledgments}

The authors express their thanks to Dr. Anjani Devi, Chairperson, Kalyani Maternity Hospital, Hanamkonda and to Prof.Y. Madhusudhan Rao, Director, Vaagdevi Group of Pharmacy Colleges for their timely support in accomplishment of this clinical study. The study received no funding or grants and was carried out without any financial support.

\section{Conflict of interest}

The authors have no conflict of interests related to this publication.

\section{Author contributions}

Manuscript writing (KA, AVK), figures and tables preparing (LK), review of the manuscript and its critical appraisal against the research objectives and its protocol (BS, SG).

\section{References}

[1] Wang C. The relationship between T2DM and related thyroid diseases. J Diabetes Res 2013:390534. doi:10.1155/2013/390534.

[2] Hage M, Zantout MS, Azar ST. Thyroid disorders and diabetes mellitus. J Thyroid Res 2011:439463. doi:10.4061/2011/439463.

[3] Kalra S, Unnikrishnan AG, Sahay R. The hypoglycemic side of hypothyroidism. Indian J Endocrinol Metab 2014;18(1):1-3. doi:10.4103/ 2230-8210.126517.

[4] Saluja M, Pyarsabadi P, Jelia S, Chittora S, Swami Y, Vimlani H. Study of thyroid dysfunction in metabolic syndrome and association with its components. Current Medicine Research and Practice 2018;8(1):37. doi:10.1016/j.cmrp.2017.11.010.

[5] Mouradian M, Abourizk N. Diabetes mellitus and thyroid disease. Diabetes Care 1983;6(5):512-520. doi:10.2337/diacare.6.5.512.

[6] Ozair M, Noor S, Raghav A, Siddiqi SS, Chugtai AM, Ahmad J. Prevalence of thyroid disorders in North Indian type 2 diabetic subjects: A cross-sectional study. Diabetes Metab Syndr 2018;12(3):301-304. doi:10.1016/j.dsx.2017.12.016.

[7] Hage M, Zantout MS, Azar ST. Thyroid disorders and diabetes mellitus-a review. J Thyroid Res 2011;2011:439463. doi:10.4061/
Gurunath S. et al: Association between T2DM and hypothyroidism

2011/439463.

[8] Kumar A. Recurrent hypoglycemia in type 2 diabetic patient due to hypothyroidism. J Diabetes Metab Disord 2015;14:13. doi:10.1186/ s40200-015-0149-y.

[9] Delarue J, Magnan C. Free fatty acids and insulin resistance. Curr Opin Clin Nutr Metab Care 2007;10(2):142-148. doi:10.1097/ MCO.0b013e328042ba90.

[10] Wang C. The relationship between type 2 diabetes mellitus and related thyroid diseases. J Diabetes Res 2013;2013:390534. doi:10.1155/2013/390534.

[11] Johnson JL. Diabetes control in thyroid disease. Diabetes Spectrum 2006;19(3):148-153. doi:10.2337/diaspect.19.3.148.

[12] Boden G. Interaction between free fatty acids and glucose metabolism. Curr Opin Clin Nutr Metab Care 2002;5(5):545-549. doi:10.1097/00075197-200209000-00014.

[13] Acharya A, Shah PB, Chitkara E, Shrestha S. Evaluation of thyroid hormone levels in patients with type 2 diabetes mellitus as compared to normal individuals in Nepal. International Journal of Health Sciences and Research 2017;7:79-85.

[14] Tuo Y, Wang D, Li S, Chen C. Long-term exposure of INS-1 rat insulinoma cells to linoleic acid and glucose in vitro affects cell viability and function through mitochondrial-mediated pathways. Endocrine 2011;39(2):128-138. doi:10.1007/s12020-010-9432-3.

[15] Neeraj Tewari. Incidence of hypothyroidism in adults: An epidemiological study in eight cities of India. Indian J Endocrinol Metab 2014;18(1):116. doi:10.4103/2230-8210.126590.

[16] Khurana A, Dhoat P, Jain G. Prevalence of thyroid disorders in patients of type 2 diabetes mellitus. Indian Academy of Clinical Medicine 2016;17(1):12-15.

[17] Centeno Maxzud M, Gómez Rasjido L, Fregenal M, Arias Calafiore $F$, Córdoba Lanus $M$, Urso M, Luciardi H. Prevalence of thyroid dysfunction in patients with type 2 diabetes mellitus. Medicina (B Aires) 2016;76(6):355-358.

[18] Khan NZ, Muttalib MA, Sultana GS, Mishu FA, Nesa A. Study of thyroid disorders among type 2 diabetic patients attending a tertiary care hospital. Mymensingh Med J 2017;26(4):874-878.

[19] Wang C. The relationship between type 2 diabetes mellitus and related thyroid diseases. J Diabetes Res 2013;2013:390534. doi:10.1155/2013/390534.

[20] Ravishankar S. N, Champakamalini, Venkatesh, Mohsin. A prospective study of thyroid dysfunction in patients with type 2 diabetes in general population. Archives of Medicine 2013;5(1):2. doi:10.3823/105.

[21] Unnikrishnan AG, Kalra S, Sahay RK, Bantwal G, John M, Tewari N. Prevalence of hypothyoidism in adults. An epidemiological study in eight cities of India. Indian J Endocrinol Metab 2013;17(4):647-652. doi:10.4103/2230-8210.113755.

[22] Prasad K, Singh S. Incidence of thyroid disorders amongst patients with diabetes- a hospital based study. International Journal of Contemporary Medical Research 2017;4(7):1497-1499.

[23] Rai S, Ashok Kumar J, Prajna K, Shetty SK, Rai T, Shrinidhi, et al. Thy roid function in T2DM and in diabetic nephropathy. J Clin Diagn Res 2013;7(8):1583-1585. doi:10.7860/JCDR/2013/6216.3299.

[24] Jali MV, Kambar S, Jali SM, Pawar N, Nalawade P. Prevalence of thyroid dysfunction among type 2 diabetes mellitus patients. Diabetes Metab Syndr 2017(Suppl 1):S105-S108. doi:10.1016/j.dsx.2016.12.017.

[25] Khatiwada S, Sah SK, Kc R, Baral N, Lamsal M. Thyroid dysfunction in metabolic syndrome patients and its relationship with components of metabolic syndrome. Clin Diabetes Endocrinol 2016;2:3. doi:10.1186/s40842-016-0021-0. 